\title{
The Outcome of Surgery in Patients with Growth Hormone Secreting Adenomas: Early Surgical Intervention is Essential for Improving the Surgical Cure Rate
}

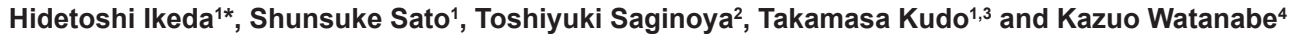 \\ 1Research Institute for Pituitary Diseases, Southern Tohoku General Hospital, Koriyama, Fukushima, Japan \\ 2Department of Radiology, Southern Tohoku General Hospital, Koriyama, Fukushima, Japan \\ 3Division of Nephrology, Endocrinology and Vascular Medicine, Tohoku University Graduate School of Medicine, Sendai, Miyagi, Japan \\ 4Research Institute for Neuroscience, Southern Tohoku General Hospital, Koriyama, Fukushima, Japan
}

\begin{abstract}
Object: During the last 10 years, 129 consecutive transsphenoidal operations for growth hormone $(\mathrm{GH})$ secreting pituitary adenomas have been carried out. In the present study, 120 of the patients involved in these operations have been reviewed to evaluate the accuracy of diagnosis of adenoma, the cure rate, and to ascertain the favorable surgical effects of early surgical intervention.
\end{abstract}

Methods: A total of $120 \mathrm{GH}$ secreting adenomas, including 95 patients with typical acromegaly and 25 patients with no apparent clinical features of acromegaly, were evaluated. The pituitary tumors were investigated by means of Magnetic Resonance (MR) imaging at $1.5 \mathrm{~T}$ and $3 \mathrm{~T}$, composite $3 \mathrm{~T}-\mathrm{MRI}$ and $\left[{ }^{11} \mathrm{C}\right]$ Methionine-positron Emission Tomography (MET-PET) imaging and histological and immunohistochemical staining. The modern remission criteria (a glucose suppressed [nadir] GH level of less than $1.0 \mathrm{ng} / \mathrm{ml}$, a random GH level of $2.5 \mathrm{ng} / \mathrm{ml}$ or less, and a normal sex and age adjusted insulin-like growth factor-1 [IGF-1] level) were used. To assess remission we examined these criteria at 1,6 and 12 months after surgery.

Results: The diagnostic accuracy of 3T-MRI and composite MET-PET fusion 3T- MRI in localizing adenoma was 87 and $100 \%$, respectively. The mean age of patients with pre-clinical acromegaly and acromegaly was 37.3 and 51.1 years, respectively. The mean preoperative $\mathrm{GH}$ levels in these patients were 4.85 and $34.7 \mathrm{ng} / \mathrm{ml}$ for preclinical acromegaly and acromegaly, respectively. The mean preoperative IGF-1 levels for pre-clinical acromegaly and acromegaly were 323 and $822 \mathrm{ng} / \mathrm{ml}$, respectively. The mean volume of the adenoma in patients with pre-clinical acromegaly and acromegaly were 1.26 and $3.84 \mathrm{~cm}^{3}$, respectively. There was a significant difference $(P<0.0001)$ between the pre-clinical acromegaly and acromegaly groups in terms of age, GH and IGF-1 levels, and tumor volume. The overall cure rate for growth hormone-secreting adenoma was $73 \%$ using modern criteria; the surgical cure rate for acromegaly patients was $65 \%$, while the surgical cure rate for patients without obvious clinical features of acromegaly was $100 \%$. Patients with pre-clinical acromegaly had a tendency to have smaller sized adenomas, a younger age, lower GH and IGF-1 levels, and a better surgical cure rate relative to patients with typical acromegaly. No complications such as carotid artery injury, hypothalamic injury, cerebrospinal fluid fistula or permanent diabetes insipidus occurred. Only one acromegaly patient had hypopituitarism after surgery.

Conclusions: Early detection of $\mathrm{GH}$ secreting adenoma is important since early surgical intervention can achieve a $100 \%$ cure rate without serious complications.

Keywords: GH adenoma; Transsphenoidal surgery; MET-PET; 3T-MRI; Cure rate; Pre-clinical acromegaly

\section{Introduction}

GH secreting pituitary adenoma is hard to diagnose in the early stages because of Growth Hormone (GH) excess and slow progression. The disease is frequently missed for many years until changes in external body features, especially the face, become noticeable. Therefore, diagnosis of the disease is delayed for approximately 2.5-10 years (mean, 8 years) from onset of symptoms [1]. Furthermore; acromegaly is associated with a twofold or threefold increase in patient mortality, and a reduced life expectancy of 10 to 15 years relative to the general population. This is due to the presence of other diseases such as diabetes mellitus, high blood pressure, hyperlipidemia, cardiovascular and respiratory disease and neoplastic complications [1-4]. The duration of disease is one of the prognostic factors. An early diagnosis of the disease is mandatory, although none of these symptoms is sufficiently sensitive, especially during the early stages of the disease. Hence, establishing guidelines for the early diagnosis or assessment of the disease activity of acromegaly is a major challenge [5-7].

In the current study, we retrospectively surveyed the surgical results of patients diagnosed with GH secreting adenoma over the past
10 years, paying special attention to the relationship between the early detection of the GH secreting adenoma and surgical outcome.

\section{Methods}

\section{Patient population}

During the last 10 years, a total of 129 patients with GH secreting adenoma underwent transsphenoidal surgery that was initially performed by one of the authors (H.I.) in the Ohara Medical Center

${ }^{*}$ Corresponding author: Hidetoshi Ikeda, M.D., Ph.D., 7-115 Yatsuyamada Research Institute for Pituitary Diseases, Southern Tohoku General Hospital Koriyama, Fukushima 963-8563, Japan, Tel: +81-24-934-5322; Fax: +81-24-9225320; E-mail: ikeda@nsg.med.tohoku.ac.jp

Received September 12, 2012; Accepted September 27, 2012; Published September 29, 2012

Citation: Ikeda H, Sato S, Saginoya T, Kudo T, Watanabe K (2012) The Outcome of Surgery in Patients with Growth Hormone Secreting Adenomas: Early Surgical Intervention is Essential for Improving the Surgical Cure Rate. J Metabolic Synd S2:001. doi:10.4172/2167-0943.S2-001

Copyright: $\odot 2012$ lkeda H, et al. This is an open-access article distributed under the terms of the Creative Commons Attribution License, which permits unrestricted use, distribution, and reproduction in any medium, provided the original author and source are credited. 
and Southern Tohoku General Hospital between 2002 and 2011. The follow-up period was more than 1 year. Nine out of the 129 patients were missed from follow-up; therefore we included 120 cases in this study. Of these patients, 51 were male and 69 were female. The patient's mean age was 48.3 years (range $13-73$ years). Of the 120 patients, there were 95 cases of "overt" acromegaly. The remaining 25 patients had no apparent clinical characteristics of acromegaly (i.e. pre-clinical acromegaly) such as obvious cheekbones, bulging forehead, enlarged jaw, and soft tissue swelling resulting in enlargement of the hands, feet, nose, lips $[1,6]$.

The present study was conducted in accordance with the declaration of Helsinki 1964. Informed consent was obtained from all patients.

\section{Magnetic resonance imaging}

The size and extent of the tumor were determined using Magnetic Resonance (MR) images obtained with a $1.5 \mathrm{~T}$ system (Magnetome: Siemens, Erlangen, Germany) and a 3.0T MR unit (Signa HDx: General Electric, Fairfield, Connecticut, USA). T1-and T2-weighted thick sagittal and coronal spin-echo MR images were obtained as 2 to $3 \mathrm{~mm}$-thick slices. Additional T1-weighted axial, sagittal and coronal MR images (500/15 ms) were obtained immediately after injection of $0.1 \mathrm{mmol} / \mathrm{kg}$ gadolinium- diethylenetriaminepenta-acetic acid (Schering, Berlin, Germany). Images were reconstructed by means of two-dimensional Fourier transformation on a $256 \times 256$ image matrix. The tumor size and cavernous sinus invasion were evaluated on axial, sagittal and coronal images with gadolinium enhancement. Tumor volume was measured using a reliable empirical formula for volume estimation published by Petersen et al. [8].

Follow-up MR imaging and hormonal examinations were carried out to detect recurrence of the tumor every 6 months after surgery.

\section{Positron emission tomography imaging}

As of 2008, the Positron Emission Tomography-Computerized Tomography (PET-CT) studies were undertaken using the Discovery LS (General Electric, Fairfield, Connecticut, USA) in all cases. The PET machine used bismuth germanium oxide crystals. All patients fasted before the procedure and received intravenous injections of $\left[{ }^{11} \mathrm{C}\right]$ methionine $(\mathrm{MET})(5.6 \mathrm{MBq} \times$ body weight [dose range, 225.7-558.3 $\mathrm{MBq}$, i.e. 6.1-15.1 mCi]). PET scans of $10 \mathrm{~min}$ duration were obtained starting at $20 \mathrm{~min}$ after injection. At $1 \mathrm{~h}$ after the MET injection all patients received intravenous injection of Fludeoxyglucose (FDG) (3.7 $\mathrm{MBq} \times$ body weight [dose range, $155-269.5 \mathrm{MBq}$, i.e. 4.2-7.3 $\mathrm{mCi}$ ]). PET scans of 10 min duration were obtained starting at $60 \mathrm{~min}$ after injection. The MET/FDG-PET procedure was performed in 3D mode, which provided a set of 35 planes with a section thickness of about 4.1 $\mathrm{mm}$. Uptake of FDG and MET during PET scanning was evaluated using a Standardized Uptake Value (SUV) max.

\section{Image post-processing}

The MR and PET images were co-registered to Gd enhanced T1weighted images and T2-weighted images using the software workstation (Advantage Windows, General Electric, Fairfield, Connecticut, USA). For co-registration between MR images and PET images, MR imaging data were first reconstructed based on the CT skull shape ascertained using PET-CT. This procedure was performed based on anatomical landmarks, such as optic nerves, the internal occipital protuberance, and the vestibular cochlear nerve. Then the MR images were fused to the PET scan. Thereafter, the PET scans that had been overlaid onto the MR images were automatically viewed [9].

\section{Laboratory evaluation}

The endocrine status of the patients were evaluated before and after surgery by measurement of serum levels of growth hormone and insulin-like growth factor-1 (IGF-1), prolactin, adrenocorticotropic hormone and cortisol, thyroid-stimulating hormone, free-T3 and free-T4, follicle-stimulating hormone, luteinizing hormone, estradiol and testosterone [10]. The secretary status of the tumor and preoperative evaluation of the baseline pituitary function were established. Precise endocrinological tests were carried out using the GH suppression test as determined using the 75 g-oGTT loading test and the TRH/ LHRH loading test, to examine the paradoxical response to GH. These stimulatory and inhibitory tests were not indicated in patients with severe diabetes mellitus or with large pituitary adenoma for fear of pituitary apoplexy.

Those patients who had glucose suppressed (nadir) GH level of less than $1.0 \mathrm{ng} / \mathrm{ml}$, a normal sex-and age adjusted IGF-1 level and postoperative random $\mathrm{GH}$ levels of $2.5 \mathrm{ng} / \mathrm{ml}$ or less, were defined as being in remission [11]. To identify remission we examined these criteria at 1, 6 and 12 months after surgery.

\section{Pathological examination}

Surgical specimens were fixed in $10 \%$ neutral buffered formalin and embedded in paraffin. $3 \mu \mathrm{m}$ thick slices were prepared and stained with $\mathrm{H} \& \mathrm{E}$. Immunohistochemical staining was performed by means of the avidin-biotin complex method using the following antibodies: polyclonal adrenocorticotropic hormone (DAKO, Glostrup, Denmark); polyclonal growth hormone (DAKO); polyclonal prolactin (DAKO, Glostrup, Denmark); monoclonal thyroid-stimulating hormone- $\beta$ (Neo markers); monoclonal luteinizing hormone- $\beta$ (Cosmo Bio Co Ltd, Tokyo, Japan); monoclonal follicle-stimulating hormone- $\beta$ (Cosmo Bio Co. Ltd); and polyclonal $\alpha$-subunit (DAKO).

\section{Statistical analysis}

Since none of the parameters showed a normal distribution, Spearman's rank correlation test was used. A P value of $<0.05$ was taken to be statistically significant. To compare the differences between two independent groups, where each group showed a normal distribution, Welch's $t$-test was used.

\section{Results}

\section{Differences in clinical factors between acromegaly and pre- clinical acromegaly}

Among 120 patients with GH secreting adenomas, 87 patients fulfilled the current remission criteria. Thus, the overall cure rate was $73 \%$. The remission rate for acromegaly $(\mathrm{N}=95)$ was $65 \%$, while the remission rate for sub-clinical acromegaly $(\mathrm{N}=25)$ was $100 \%$. Alternatively, using old remission criteria (nadir $\mathrm{GH}<2$ ), the overall remission rate was found to be $81 \%$.

In patients with pre-clinical acromegaly and acromegaly the mean ages were 37.3 and 51.1 years, respectively; the mean preoperative $\mathrm{GH}$ levels were 4.85 and $34.7 \mathrm{ng} / \mathrm{ml}$, respectively; the mean preoperative IGF1 levels were 323 and $822 \mathrm{ng} / \mathrm{ml}$, respectively; and the mean volume of the adenomas was 1.26 and $3.84 \mathrm{~cm}^{3}$, respectively. There was a significant difference between the pre-clinical acromegaly group and the acromegaly group with regard to age, GH value, IGF-1 value and tumor volume (Table 1). Thus, patients with pre-clinical acromegaly, that is to say adenomas which were found in the early stage, showed a tendency to have smaller sized tumors, a younger age, a lower value of GH and IGF-1 and a better surgical cure rate relative to patients with acromegaly. 
Citation: Ikeda H, Sato S, Saginoya T, Kudo T, Watanabe K (2012) The Outcome of Surgery in Patients with Growth Hormone Secreting Adenomas: Early Surgical Intervention is Essential for Improving the Surgical Cure Rate. J Metabolic Synd S2:001. doi:10.4172/2167-0943.S2-001

Page 3 of 4

\section{Diagnostic sensitivity of MRI and MET-PET}

The diagnostic sensitivity of MRI was shown to be $87 \%$ (i.e. 105 patients out of 120 patients showed positive findings), and that of METPET fusion MRI 100\% (i.e. all of the 47 patients examined showed positive findings). Thus the diagnostic accuracy of MET-PET was perfect and far superior to the diagnostic accuracy of MRI (Figure 1).

\section{Pathological results}

In our current series, 115 out of 120 tumors were subjected to pathological evaluation. A variety of combinations of hormone production were observed (Figure 1). All of the adenomas, needless to say, showed strong positive immune reactivity for GH. In addition, Prolactin (PRL) was the second most frequent (79\%) immune-positive hormone, followed by FSH beta (33\%), ACTH (32\%), LH beta (29\%) and TSH beta (23\%). The mean number of hormones produced from adenomas in patients with acromegaly was 2.8 , while in patients with pre-clinical acromegaly it was 3.6. There was a significant difference $(\mathrm{P}=0.019$, Welch's $t$ test $)$ in the number of hormones produced between these two patient groups. The adenoma in patients with pre-clinical acromegaly tended to produce a higher number of different hormones.

\begin{tabular}{|l|l|l|l|}
\hline & $\begin{array}{l}\text { Pre-clinical Acromegaly } \\
\mathbf{( N = 2 5 )}\end{array}$ & $\begin{array}{l}\text { Acromegaly } \\
(\mathbf{N}=\mathbf{9 5})\end{array}$ & differences \\
\hline Age & 37.3 & 51.1 & $\mathrm{P}=0.000315$ \\
\hline GH value $(\mathbf{n g} / \mathbf{m l})$ & 4.85 & 34.7 & $\mathrm{P}=0.0000001$ \\
\hline IGF-1 value $(\mathbf{n g} / \mathbf{m l})$ & 323 & 822 & $\mathrm{P}=0.00000003$ \\
\hline Adenoma volume(cm3) & 1.26 & 3.84 & $\mathrm{P}=0.000005$ \\
\hline Cure rate & $100 \%$ & $65 \%$ & \\
\hline
\end{tabular}

Table 1: Comparison of clinical factors between acromegaly and pre-clinical acromegaly.

\section{Complications of surgical management}

Hypopituitarism after surgery occurred in one $(0.8 \%)$ out of 120 patients, and no patient suffered from permanent Diabetes Insipidus (DI). There was no mortality and morbidity, and none of the patient had a Cerebrospinal Fluid (CSF) fistula.

\section{Adjuvant therapy}

Among the 33 patients who did not fulfill the modern cure criteria, 28 patients underwent gamma knife radiotherapy [12], 12 as a rule, at 1 year after operation. Two patients underwent conventional radiotherapy because the residual tumor was too large for gamma knife treatment. Two patients received sandostatin LAR treatment. Four patients were placed under strict observation because the nadir in GH level after 75 g-oGTT was borderline (1-2 $\mathrm{ng} / \mathrm{ml})$.

\section{Discussion}

Our data evaluation revealed that it is important to diagnose $\mathrm{GH}$ secreting adenoma as early as possible because this contributes to an improved surgical cure rate, resulting in the prevention of progressive organ failure. At the same time adverse effects caused by the surgical procedures were minimal because most of the early stage adenoma corresponded to Knosp grade 0-1. Therefore, there was no need for manipulation in the cavernous sinus, resulting in a reduced risk factor for surgery.

The overall cure rates obtained in studies involving several large series of patients with acromegaly treated by transsphenoidal surgery using modern cure criteria were reported as follows: $67 \%(\mathrm{~N}=117$, Kreutzer et al. [13]), 57\% ( $\mathrm{N}=506$, Nomikos et al. [14]), 52\% $(\mathrm{N}=$ 103, Beauregard et al. [15]) and $64 \%(\mathrm{~N}=160$, Giustina et al. [11]).
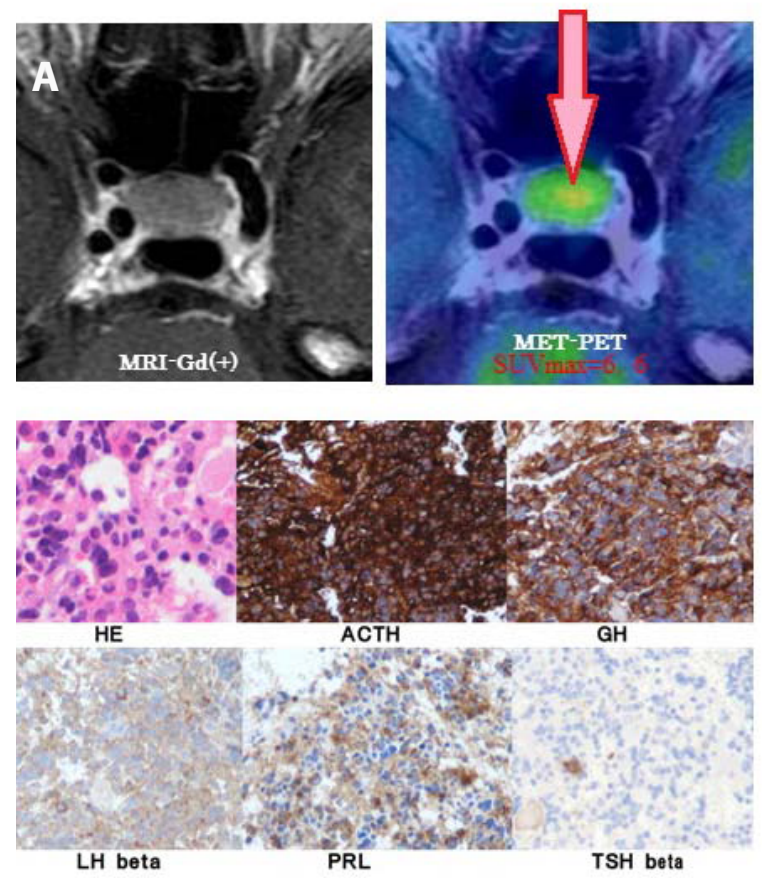
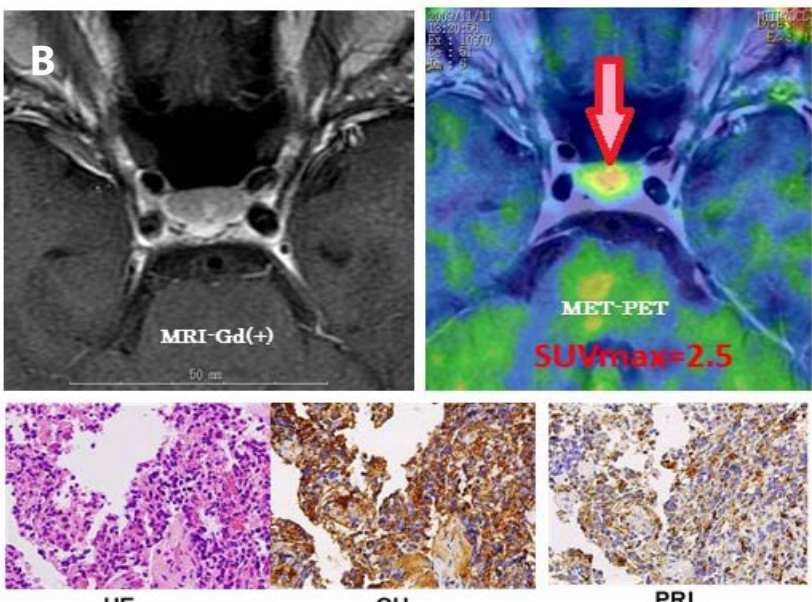

PRL

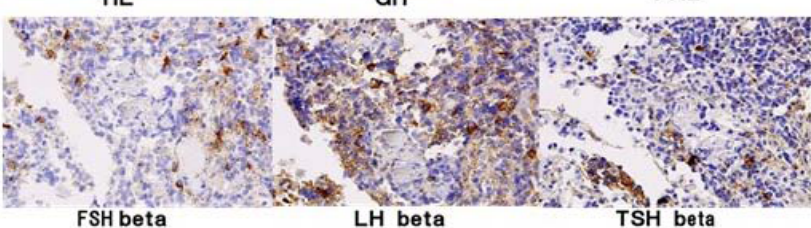

Figure 1: (a) A 33 year old female with pre-acromegaly. Although 3T-MRI could not detect the adenoma, MET-PET fusion MRI successfully demonstrated its localization. The MET SUV max was 6. 6. The arrow indicates high uptake of methionine. Pathological analysis indicated intense immunoreactivity for GH and ACTH. In addition, LH beta, PRL and TSH beta were also immune-positive in the same adenoma tissue.

(b) A 47 year old female with pre-acromegaly. Although 3T-MRI could not detect the adenoma, MET-PET fusion MRI successfully demonstrated its localization. The MET SUV max was 2.5. The arrow indicates high uptake of methionine. Pathological analysis revealed intense immunoreactivity for GH. In addition PRL, FSH beta, $\mathrm{LH}$ beta and TSH beta were also immune-positive in the same adenoma tissue. 
Citation: Ikeda H, Sato S, Saginoya T, Kudo T, Watanabe K (2012) The Outcome of Surgery in Patients with Growth Hormone Secreting Adenomas: Early Surgical Intervention is Essential for Improving the Surgical Cure Rate. J Metabolic Synd S2:001. doi:10.4172/2167-0943.S2-001

Page 4 of 4

The overall cure rate for GH secreting adenoma in our study was $73 \%$ using modern cure criteria [16] and $81 \%$ using the previously reported cure rate. Our surgical cure rate was much higher than those reported in previous studies, probably because early detection and early surgical intervention contributed to an improvement in cure rate.

For the detection and delineation of tumor location, especially in the case of microadenoma, the diagnostic accuracy of superconductive MR imaging has been reported to be only 10 to $40 \%$, with $33 \%$ falsenegatives and $20 \%$ false-positives [15-18]. In general, the use of dynamic MR imaging is considered to improve diagnostic accuracy. However, Tabarin et al. [19] reported on the rates of accurate diagnosis with MRI and dynamic MRI using 1.0-T MRI. They indicated that true positivity was $53 \%$ for MRI and $67 \%$ for dynamic MRI, illustrating that there was no significant difference between the diagnostic accuracy of these two imaging techniques.

Conventional imaging has failed to diagnose early stage $\mathrm{GH}$ secreting adenoma. However, MET-PET fusion MRI has enabled the detection of adenoma in all patients with abnormal GH dynamics that have been examined [15]. Pre-clinical Cushing's adenoma was efficiently detected using MET-PET fusion MRI; [16,17] similarly, pre-clinical acromegaly was also detected by MET-PET fusion MRI. The reason why MET-PET has a high sensitivity in the detection of microadenoma is attributed to the following factors: (1) both GH adenoma and ACTH adenoma have high cellularity relative to normal anterior pituitary tissue; (2) almost all of the cells in these two types of adenoma show strong positive immunoreactivity for either GH or ACTH; (3) electron microscopy studies have shown that both ACTH adenoma and GH adenoma have densely packed secretary granules in their cytoplasm; and (4) both adenomas from pre-clinical Cushing's disease and preclinical acromegaly exhibit multiple hormone production [20]. In the light of these findings it is clear that amino acid metabolism in both ACTH adenoma and GH adenoma is extremely active, thus accounting for the high accumulation of methionine during MET-PET.

The goal of the overall management of acromegaly should be to provide patients with the most effective means of long-term control of this benign but potentially disabling disease. For this purpose, early surgical treatment of acromegaly promises a high cure rate and minimal adverse surgical effects.

\section{Conclusions}

Early-stage GH secreting adenomas are inevitably microadenoma, and as a result the rate of detection of these tumors using superconductive MR imaging is unsatisfactory.

However, the MET-PET fusion 3T-MRI method provides a far higher sensitivity (100\%) in delineating GH secreting adenoma and determining its location. We emphasize the usefulness of MET-PET fusion MRI in diagnosing GH secreting adenoma, if the presence of a GH producing tumor is suspected from an endocrinological point of view. This in turn contributes to the early diagnosis and high surgical cure rate of GH-secreting adenoma.

\section{References}

1. Ben-Shlomo A, Melmed S (2008) Acromegaly. Endocrinol Metab Clin North Am 37: $101-122$

2. Baldys-Waligorska A, Krzentowska A, Golkowski F, Sokolowski G, HubalewskaDydejczyk A (2010) The prevalence of benign and malignant neoplasms in acromegalic patients. Endokrynol Pol 61: 29-34.

3. Brooke AM, Drake WM (2007) Serum IGF-levels in the diagnosis and monitoring of acromegaly. Pituitary 10: 173-179.

4. Colao A, Ferone D, Marzullo P, Lombardi G (2004) Systemic complications of acromegaly: epidemiology, pathogenesis, and management. Endocr Rev 25: 102-152.

5. Bergstrom M, Muhr C, Lundberg PO, Langstrom B (1991) PET as a tool in the clinical evaluation of pituitary adenomas. J Nucl Med 32: 610-615.

6. Chanson P, Salenave S, Kamenicky P, Cazabat L, Young J (2009) Pituitary tumours: acromegaly. Best Pract Res Clin Endocrinol Metab 23: 555-574.

7. De Souza B, Brunetti A, Fulham MJ, Brooks RA, DeMichele D, et al. (1990) Pituitary microadenomas: a PET study. Radiology 177: 39-44.

8. Peterson OF, Esperson JO (1984) Extradural hematomas: measurement of size by volume summation on CT scanning. Neuroradiology 26: 363-367.

9. Ikeda $\mathrm{H}$, Abe T, Watanabe $\mathrm{K}$ (2010) Usefulness of composite methioninepositron emission tomography/3.0-tesla magnetic resonance imaging to detect the localization and extent of early-stage Cushing adenoma. J Neurosurg 112 750-755.

10. Losa M, Oeckler R, Schopohl J, Muller OA, Alba-Lopez J, et al. ( 1989) Evaluation of selective transsphenoidal adenomectomy by endocrinological testing and somatomedin-C measurement in acromegaly. J Neurosurg 70 : 561-567.

11. Giustina A, Barkan A, Casanueva FF, Cavagnini F, Frohman L, et al. (2000) Criteria for cure of Acromegaly: A consensus statement. J Clin Endocrinol Metab 85: 526-529.

12. Ikeda $\mathrm{H}$, Jokura $\mathrm{H}$, Yoshimoto $\mathrm{T}$ (2001) Transsphenoidal surgery and adjuvant gamma knife treatment for growth hormone-secreting pituitary adenoma. $J$ Neurosurg 95: 285-291.

13. Kreutzer J, Vance ML, Lopes MB, Laws ER Jr (2001) Surgical management of GH-secreting pituitary adenomas: an Outcome study using modern remission criteria. J Clin Endocrinol Metab 86: 4072-4077.

14. Nomikos P, Buchfelder M, Fahlbusch R (2005) The outcome of surgery in 668 patients with acromegaly using current criteria of biochemical 'cure'. Eur $J$ Endocrinol 152: 3790-387.

15. Beauregard C, Truong U, Hardy J, Serri O (2003) Long-term outcome and mortality after transsphenoidal adenomectomy for acromegaly. Clin Endocrino (Oxf) 58: 86-91.

16. Bonneville JF, Bonneville F, Cattin F ( 2005) Magnetic resonance imaging of pituitary adenomas. Eur Radiol 15: 543-548.

17. Ikeda H (2011) Diagnosis of Cushing's disease. In: Berhardt LV (Ed.), Advances in Medicine and Biology. New York: Nova Science Publishers.

18. Ikeda H, Sato S, Saginoya S, Watanabe K (2011) MET-PET images of patients with abnormal GH secretary dynamics. Endocr Pathol 23: 70-71.

19. Tabarin A, Laurent F, Catargi B, Olivier-Puel F, Lescene R, et al. (1998) Comparative evaluation of conventional and dynamic magnetic resonance imaging of the pituitary gland for the diagnosis of Cushing's disease. Clin Endocrinol (Oxf) 49: 293-300

20. Ikeda $\mathrm{H}$, Watanabe $\mathrm{K}$ (2011) Multifunctional cells in human pituitary are involved in pituitary tumor genesis-pathological analysis of microadenoma in the early stage diagnosed by 3T-MRI fusion MET-PET scans. Endocr Rev 32: 1-425.
This article was originally published in a special issue, Pathophysiology and Treatment of Acromegaly handled by Editor(s). Dr. Hidetoshi Ikeda, Southern Tohoku General Hospital, Japan 